\title{
De la seguridad al riesgo psicosocial en el trabajo en la legislación colombiana de salud ocupacional ${ }^{\star}$
}

\author{
From safety to psychosocial risk at work in Colombian legislation \\ of occupational health \\ Da segurança ao risco psicossocial no trabalho na legislação colombiana \\ de saúde ocupacional
}

\author{
FÁTIMA DíAZ BAMBULA ${ }^{*}$ \\ ERICO ReNTERÍA Pérez ${ }^{* * *}$
}

FeCha de ReCePCión: 2 de Julio de 2016. FeCha de ACEPTACIÓn: 4 de febrero de 2017

Para citar este artículo: Díaz, F., E Rentería, E. (2017). De la seguridad al riesgo psicosocial en el trabajo en la legislación colombiana de salud ocupacional. Estudios Socio-Jurídicos, 19(2), 129-155. Doi: xxxxxxx

\section{RESUMEN}

Ante el hecho de que en Colombia el ejercicio de los psicólogos en el campo de la salud ocupacional ha sido normativizado, es importante conocer y reflexionar sobre la legislación, considerando que las primeras leyes abordaron esta problemática desde la seguridad y, en la actualidad, se administra la salud desde la gestión del riesgo laboral. Este artículo presenta la síntesis de una exhaustiva revisión del desarrollo de la legislación sobre salud ocupacional en Colombia, mostrando cómo la salud en el trabajo fue inicialmente abordada desde los accidentes pasando al riesgo, concepto que da espacio para un reconocimiento desde la normativa de fenómenos psicosociales como el estrés y el síndrome de burnout. Se concluye que la relación existente entre el trabajo y la salud ha sido y hace parte de la relación ontológica persona-trabajo a lo largo de la historia, la seguridad en el trabajo

* Esta investigación fue presentada en su fase inicial dentro del simposio "Psicología Ocupacional: Avances en su Abordaje Conceptual y Metodológico" en el Congreso Colombiano de Psicología 2015 y la 1. a Conferencia Regional Latinoamericana de Psicología 2015 realizado en Armenia, Colombia.

** Magíster en Psicología de la Universidad del Valle. Candidata a doctora en Psicología de la Universidad del Valle. Docente del Instituto de Psicología de la Universidad del Valle, Cali, Colombia. Integrante del grupo de investigación de Psicología del Trabajo y las Organizaciones. Correo electrónico: fatima.diaz@correounivalle.edu.co

*** Doctor en Psicología de la Universidad de São Paulo. Magíster de la Pontificia Universidad Católica de São Paulo. Docente del Instituto de Psicología Universidad del Valle, Cali, Colombia. Director del grupo de investigación de Psicología del Trabajo y las Organizaciones. Correo electrónico: erico.renteria@correounivalle.edu.co 
trae consigo un ejercicio de poder y de orden social y, por último, que el ejercicio de los psicólogos ha sido normativizado y, por esto, tecnificado.

Palabras claves: legislación, Colombia, psicología de salud ocupacional, riesgo y seguridad

\begin{abstract}
The professional practice of occupational health psychology in Colombia has been legislated since 2008. Originally, Colombian law had been focused on safety and health; currently the emphasis is the management of risk, phenomena that in our country must be treated only by occupational health psychologists licensed for this. That gives space to recognize the normative psychosocial phenomena such as stress and burnout syndrome. It is important that psychologists know and reflect on the laws governing the practice. This review seeks to do an approach to reflect the implications of these regulations at the level of security, risk, social order, and logic of production.
\end{abstract}

Keyeords: Legislation, Colombia, occupational health psychology, risk and safety

\title{
RESUMO
}

Ante o facto de que na Colômbia o exercício dos psicólogos no campo da saúde ocupacional tem sido normatizado, é importante conhecer e refletir sobe a legislação, considerando que as primeiras leis abordaram esta problemática desde a segurança e, na atualidade, se administra a saúde desde a gestão do risco laboral. Este artigo apresenta a síntese de uma exaustiva revisão do desenvolvimento da legislação sobre saúde ocupacional na Colômbia, mostrando como a saúde no trabalho foi inicialmente abordada desde os acidentes, passando ao risco, conceito que dá espaço para um reconhecimento desde a normativa de fenômenos psicossociais como o stress e o síndrome de burnout. Conclui-se que a relação existente entre o trabalho e a saúde tem sido e faz parte da relação ontológica pessoa-trabalho ao longo da história, a segurança no trabalho traz consigo um exercício de poder e de ordem social e, pelo último, que o exercício dos psicólogos tem sido normatizado e, por isso, modernizado.

Palavras-chave: legislação, Colômbia, psicologia de saúde ocupacional, risco e segurança. 


\section{Introducción}

El objeto de este artículo es mostrar un panorama de la normativa para la salud ocupacional en Colombia con atención específica a los fenómenos psicosociales relacionados. Se reflexiona sobre los conceptos de seguridad y riesgo que atraviesan la normativa; adicionalmente se plantea una discusión acerca del ejercicio de los psicólogos en el campo de la salud ocupacional, que ha sido reglamentado desde el 2008.

La estrategia metodológica utilizada para esto corresponde a una revisión sistemática y exhaustiva de las leyes colombianas relacionadas con la salud en el lugar del trabajo (Beltrán, 2005; Letelier, Manríquez E Rada, 2005; Nelson, 2002; Perestelo-Pérez, 2012; Toro, 2001). Se revisaron las páginas web del Ministerio de Salud y Protección Social y la del Ministerio de Trabajo de Colombia. Adicionalmente se hizo uso de otras fuentes que configuran la amplia literatura sobre el campo y que nutren la visión sobre la salud ocupacional, tales como artículos científicos, reportes de investigaciones, boletines jurídicos, informes de los ministerios, entre otros. La información obtenida de las normativas fue sistematizada y analizada utilizando matrices. En un primer momento las matrices contenían el nombre, el tipo (ley, decreto, resolución), el año, el objeto, los conceptos (psicosocial, riesgo, seguridad, enfermedad y accidente, entre otros) y observaciones. A partir de lo anterior, se presentan a manera de resultados por bloques temáticos los hallazgos y reflexiones derivados de las diversas fuentes analizadas.

El primer apartado corresponde al proceso normativización de la salud en el trabajo en Colombia desde 1915 hasta la fecha, seguido por la regulación del ejercicio profesional de los psicólogos; el segundo bloque aborda la discusión y reflexiones en torno a la seguridad y el orden social desde la normativa, la evolución conceptual desde el enfoque los accidentes de trabajo al de los riesgos laborales como mecanismos usados en la lógica de la producción, y la psicología en la normativa; por último se presentan las conclusiones. 


\section{Marco teórico}

El inicio de la regulación de seguridad de los trabajadores en cuanto a los accidentes de trabajo se ha venido gestando desde comienzos del siglo pasado en América Latina (Bronstein, 1998; García, 2008). Bronstein (1998), quien realizó para la Organización Internacional del Trabajo (OIT) un marco histórico sobre las reformas de la legislación laboral en América Latina, plantea la existencia de seis grandes períodos: 1) Al siglo XIX y al comienzo del siglo XX les corresponden regulaciones laborales de conformidad con las normas civiles de arrendamiento de servicios. 2) Tanto en América Latina como en Europa las primeras leyes obreras constituyen el punto de partida de la regulación laboral que consistía en normas civiles sobre el arrendamiento de servicios. En la segunda mitad del siglo XIX estas normas fueron recogidas en códigos civiles. 3) En 1917, en la Constitución mexicana, se formula la doctrina sobre el deber que tiene el Estado de legislar para proteger al trabajador; este período se prolonga hasta comienzos de los años treinta del siglo XX. 4) En 1931, con la Ley Federal del Trabajo de México y el Código de Trabajo de Chile, se inicia la consolidación y codificación de la legislación laboral que concluye treinta años después, en 1961, con el Código de Trabajo de Paraguay. 5) Fase de profundización de las garantías laborales, que concluye hacia 1974 con la Ley de Contrato de Trabajo de la Argentina. 6) En la segunda mitad de los setenta se inicia una fase de retrocesos y vaivenes, que comienza con la Ley 95 de Panamá. En ella se alternan reformas tendentes a recortar las garantías de los períodos anteriores, que son las más numerosas, con otras, las menos, que van en sentido contrario.

\subsection{El proceso normativización de la salud en el trabajo en Colombia}

A continuación se realiza un breve recuento de la historia de la legislación en materia de salud ocupacional en Colombia desde 1904.

Los inicios están vinculados con el general Rafael Uribe Uribe quien, motivado por el proceso de seguridad que se había desarrollado en Europa desde el siglo XIX, fue el primero en plantear en Colombia el tema de la seguridad en el trabajo durante una conferencia dictada en Bogotá 
en el año 1904, en la cual plantea la necesidad de dictar leyes sobre los accidentes de trabajo y sobre la protección de los niños, jóvenes y mujeres en los talleres y trabajos del campo (Cruz, 1996).

En Colombia, al igual que en el contexto internacional de comienzos del siglo XX, se vivieron luchas y protestas obreras en contra de la explotación y las precarias condiciones de vida. A partir de 1912 hubo un florecimiento del movimiento obrero en diversos sectores de la producción como la construcción de infraestructura de transporte, la industria manufacturera, las industrias extractivas, el transporte y la agricultura industrializada, entre otros. Las protestas estaban motivadas por la búsqueda de mejoras en las condiciones de trabajo y de vida, por ejemplo una mayor remuneración, asistencia médica, reducción de las jornadas laborales y mejora en las condiciones de vivienda, entre otras (Arana $\mathcal{E}$ Guerrero, 2013; Elías, 2013; Vega, 2002).

Después del asesinato de Rafael Uribe Uribe, se aprueba en el Congreso la Ley 57 de 1915, con la cual se obliga a la reparación por accidentes de trabajo a las empresas de alumbrado público, de acueductos públicos, de ferrocarriles y tranvías, de arquitectura o construcción de albañilería en que trabajen más de quince obreros, a las minas y canteras, navegación por embarcaciones mayores, industriales servidas por maquinaria con fuerza mecánica y las obras públicas nacionales y a las fábricas de licores y de fósforos (art. 10. ${ }^{\circ}$.

En el artículo 11 se encuentra, sin embargo, una prerrogativa en la cual se plantea que:

Artículo $11 .^{\circ}$ En los trabajos que dependan de empresarios, industriales o cualquiera otra clase de capitalistas cuyo capital no alcance a mil pesos $\left(\$ 1,000^{.00}\right)$ oro, no están obligados a pagar indemnización de accidentes de trabajo, sino la asistencia médica de la que habla el artículo 7 de esta Ley.

Con esto, las formas de seguridad están supeditas al número de trabajadores y al capital de la empresa, privilegiando así lugares de no responsabilidad para las empresas o patronos que afectan a trabajadores que se encuentran inmersos en esas condiciones. 
El proceso de normativizar la protección de los trabajadores continúa y en 1918 surge la Ley 46 de 1918 mediante la cual se dicta una medida de salubridad pública y se provee la existencia de habitaciones higiénicas para la clase proletaria, seguida por la Ley 37 de 1921, que establecía un seguro de vida colectivo para empleados y la Ley 10 de 1934, donde se reglamentaba la enfermedad profesional, auxilios de cesantías, vacaciones y contratación laboral. En 1934 se creó la Oficina de Medicina Laboral y en 1935 se establece la Legislación Laboral y se habla de los riesgos del trabajo. En 1938 se crean los Ministerios de Trabajo, Higiene y Previsión Social y de Economía Nacional mediante la Ley 96. En 1945 se establece la legislación sobre salud ocupacional en la cual figura la primera ley sobre normas de salud ocupacional (Ley 6 de 1945). Con esta ley comienzan a regularse las jornadas laborales, la diferencia entre salarios, el descanso dominical y auxilio de cesantía, entre otras condiciones.

Con la Ley 90 del 1946 se crea el Instituto Colombiano de Seguros Sociales y se establece el seguro social obligatorio de los trabajadores. Para 1950 se expide el Código Sustantivo del Trabajo con el cual se establecen múltiples normas relativas a la salud ocupacional como la jornada laboral, el descanso obligatorio y las prestaciones (Henao, 2010).

En la década de los sesenta la OIT asesora a Colombia para al gobierno en el montaje y la estructuración del SENA (Servicio Nacional de Aprendizaje). Según Urdaneta (1993) con la alianza entre el Ministerio de Trabajo, la Seguridad Social y de Salud y la OIT se promovieron en Colombia reglamentaciones que buscaban "garantizar un ambiente de trabajo sano y reducir así la incidencia de factores causantes de enfermedades profesionales o prevenir la ocurrencia de accidentes en los sitios de trabajo" (p. 358).

En 1964 mediante el Decreto 3170 se aprueba el Reglamento del Seguro Social obligatorio para Accidentes de Trabajo y Enfermedades Profesionales y en 1968 la protección para Accidentes de Trabajo y Enfermedades Profesionales de los Servidores del Sector Público que se empezó a manejar a través de la Caja Nacional de Previsión Social (Cajanal) (Henao, 2010).

La Ley 9 de 1979 mediante la cual se dictan medidas sanitarias se refiere en su Título III a la salud ocupacional que tiene por objeto pre- 
servar, conservar y mejorar la salud de los individuos en sus ocupaciones; plantea que se debe prevenir todo daño para la salud de las personas, derivado de las condiciones de trabajo, proteger a la persona contra los riesgos relacionados con agentes físicos, químicos, biológicos, orgánicos, mecánicos y otros que pueden afectar la salud individual o colectiva en los lugares de trabajo; que se debe eliminar o controlar los agentes nocivos para la salud en los lugares de trabajo; que se debe proteger la salud de los trabajadores y de la población contra los riesgos causados por las radiaciones y proteger a los trabajadores y a la población contra los riesgos para la salud provenientes de la producción, el almacenamiento, el transporte, el expendio y el uso o disposición de sustancias peligrosas para la salud pública.

En 1983 de acuerdo al Decreto 586 se constituye el Comité Nacional de Salud Ocupacional se establece su creación y se plantea que tiene como objetivo diseñar y coordinar permanentemente los programas de salud ocupacional. Según García (2008) este comité se encargaba de "coordinar e integrar acciones y programas, focalizar recursos, aunar esfuerzos y establecer un plan para evitar la colisión de competencias de las entidades públicas y privadas" (p. 222).

En el siguiente año mediante el Decreto 614 de 1984, se establecen nuevos parámetros para la organización de la salud ocupacional y se modifica la composición del Comité Nacional de Salud Ocupacional, estableciendo que los empleadores tienen la obligación de estructurar, desarrollar y evaluar programas integrales de salud ocupacional en las empresas. Esto conllevó a la consolidación de los Comités Paritarios de Salud Ocupacional que tienen como responsabilidad la promoción y vigilancia de las normas y reglamentos de salud ocupacional dentro de las empresas. Desde ese momento se comienzan a considerar los riesgos psicosociales. En la Resolución 1016 de 1989 con la cual se reglamenta la organización, el funcionamiento y la forma de los programas de salud ocupacional que deben desarrollar los patronos o empleadores en el país, se incluye los programas de medicina preventiva y medicina del trabajo, higiene industrial y seguridad industrial, el cual será ampliado en la Resolución 2646 de 2008 en relación con los riesgos psicosociales.

En la década de los noventa, después de la Constitución de 1991, en el marco legislativo por medio de la Ley 100 de 1993 se establece el 
Sistema de Riesgos Profesionales, el cual es definido como un conjunto de normas, entidades y procedimientos destinados a prevenir y proteger a los trabajadores de los efectos de las enfermedades y los accidentes que puedan ocurrir con ocasión o como consecuencia del trabajo y de atenderlos en estos casos. Con la expedición de la Ley 1562 de 2012 el sistema cambia a Sistema de riesgos laborales con el fin de permitir la ampliación de la cobertura de los trabajadores que deben ser afiliados al sistema y de los trabajadores informales que ahora podrán afiliarse de manera voluntaria. Se incluyó, dentro de los riesgos laborales, aquellos eventos que les sucedan a los trabajadores en ejercicio de función sindical, aún en permiso sindical.

\subsection{La regulación del ejercicio profesional de los psicólogos}

Adicionalmente se encuentran el Decreto 1295 de 1994 que regula el ejercicio profesional y la resolución 2046 de 2008 que corresponde a los factores de riesgo psicosocial y que otorga poder a los psicólogos para intervenir en este campo. El Decreto 1295 de 1994 plantea en su artículo $2 .^{\circ}$ el objetivo del sistema general de riesgos profesionales:

a. Establecer las actividades de promoción y prevención tendientes a mejorar las condiciones de trabajo y salud de la población trabajadora, protegiéndola contra los riesgos derivados de la organización del trabajo que puedan afectar la salud individual o colectiva en los lugares de trabajo tales como los físicos, químicos, biológicos, ergonómicos, psicosociales, de saneamiento y de seguridad.

En el 2006 se radica una ley contra el acoso laboral, la Ley 1010 de 2006, que:

Tiene por objeto definir, prevenir, corregir y sancionar las diversas formas de agresión, maltrato, vejámenes, trato desconsiderado y ofensivo y en general todo ultraje a la dignidad humana que se ejercen sobre quienes realizan sus actividades económicas en el contexto de una relación laboral privada o pública. 
En el 2007 el Ministerio de la Protección Social realizó la Primera Encuesta Nacional de Condiciones de Salud y Trabajo en el Sistema General de Riesgos Profesionales (I ENCST). En esa encuesta se reporta que los sectores con mayor cifra de muerte de trabajadores son el de la construcción y el de las minas y canteras, seguido por el del transporte, el de almacenamiento y comunicaciones, de electricidad, gas y agua, de agropecuaria, administración pública y defensa, de seguridad social y otras actividades de servicios comunitarios, sociales y personales (Ministerio de la Protección Social, 2007).

Esa encuesta reveló también que una tercera parte de los trabajadores manifestaron sentir altos niveles de estrés; también se encontraron niveles de cansancio y fatiga relacionados con los accidentes de trabajo, lo que mostró que los factores del entorno laboral que influyen en la salud de los trabajadores son en primer lugar "las condiciones ergonómicas (movimientos repetitivos de manos o brazos, conservar la misma postura durante toda o la mayor parte de la jornada laboral, posiciones que pueden producir cansancio o dolor)" (Ministerio de la Protección Social, 2007, p. 52) seguidas por "los agentes psicosociales relacionados con la atención de público y trabajo monótono" (Ministerio de la Protección Social, 2007, p. 52), especialmente en "centros de trabajo de las actividades económicas del comercio, actividades inmobiliarias, industria manufacturera y transporte" (Ministerio de la Protección Social, 2007, p. 102).

En la Segunda Encuesta Nacional de Condiciones de Seguridad y Salud en el Trabajo en el Sistema General de Riesgos Laborales de Colombia (II ENCSST) realizada en el 2013 se mantienen como factores de riesgo los ergonómicos y los psicosociales. Sin embargo, en esta evaluación los riesgos psicosociales, en especial la atención al público, superan a los riesgos ergonómicos, invirtiendo el orden de los factores de riesgo en relación con la I ENCST. Por otro lado en cuanto al acoso laboral se encontró que en el 2007 el $3 \%$ de los casos eran de acoso de subalternos a superiores y el 6\% de acoso por parte de superiores; para el 2013 se presenta que el $5 \%$ del acoso es ocasionado por el jefe inmediato y el $4.4 \%$ por los compañeros y subalternos.

La Resolución 2646 de 2008 tiene por objeto dar los parámetros que ayuden a establecer o identificar las responsabilidades en cuanto a 
la exposición que pueden tener los trabajadores a factores de riesgo psicosocial como producto del estrés ocupacional. Estos parámetros constituyen el marco legal para la evaluación, prevención, estudio y manejo del citado riesgo y se establecen disposiciones y se definen responsabilidades para la identificación, evaluación, prevención, intervención y el monitoreo permanente de la exposición a factores de riesgo psicosocial en el trabajo y para la determinación del origen de las patologías causadas por el estrés ocupacional.

Con esta resolución emergen con fuerza las evaluaciones, discusiones e intervenciones en relación con los factores psicosociales. Esta resolución en el capítulo I tiene un diccionario de lo que se entenderá por riesgo, trabajo, factor de riesgo, factor de riesgo psicosocial, condiciones de trabajo, estrés, experto y patologías derivadas del estrés, entre otros.

En el 2010, con la información reportada en la Primera Encuesta Nacional sobre Condiciones de Salud y Trabajo (2007) y la Resolución 2646 de 2008, el Ministerio de la Protección Social planteó la necesidad de la creación de una evaluación estandarizada de riesgos psicosociales para lo cual contrató a la Pontificia Universidad Javeriana de Bogotá. Se construyó la Batería de instrumentos para la evaluación de factores de riesgo psicosocial, teniendo en cuenta que la Resolución 2646 de 2008 que considera que los factores psicosociales deben ser evaluados objetiva y subjetivamente, utilizando instrumentos que hayan sido validados en el país. El Ministerio de la Protección Social (2010) planteó que "el alcance de esta batería de instrumentos es el de evaluar los factores de riesgo psicosociales, entendidos como las condiciones psicosociales cuya identificación y evaluación muestra efectos negativos en la salud de los trabajadores o en el trabajo" (p. 28).

En el 2003 Idrovo realizó un estudio que presenta una estimación de la incidencia de enfermedades ocupacionales en Colombia entre 1985-2000. Los resultados mostraron un incremento en la incidencia de enfermedades ocupacionales de 68063 casos en 1985 a 101645 en 2000. Idrovo (2003) plantea para ese momento que la falta de profesionales clínicos capacitados en el diagnóstico de enfermedades ocupacionales dificulta la calificación de una enfermedad como laboral al considerar mecanismos unicausales, lejanos a la multicausalidad propia de las enfermedades humanas. Según Matabanchoy (2012), la mortalidad registrada en 2003 
alcanzó una tasa de 18,29 por cada 100000 trabajadores afiliados y la tasa de enfermedades profesionales registradas en el 2004 en Colombia fue de 1,38 casos por 10000 trabajadores. En ambos casos los autores hacen referencia a enfermedades ocupacionales del orden fisiológico. La observación que hace Idrovo (2003) respecto a la multicausalidad propia de las enfermedades humanas es aún más válida en relación con lo psicosocial, dadas las características de los fenómenos de este orden. Allí se perfila claramente una tendencia hacia el síndrome de burnout.

Esta dificultad se hace más notoria cuando se define el término de enfermedad, que es entendido como alteración con causas conocidas y específicas (Cadavid, 1942). En cambio el término síndrome, una palabra de origen griego que hace referencia a una falta de curso, en términos médicos se refiere a un conjunto de síntomas característicos de una enfermedad de causa variada. Sin embargo Cadavid (1942), refiriéndose a la etimóloga del término enfermedad, hace la aclaración que se habla de síndrome en relación con las alteraciones mentales, lo que muestra que los fenómenos de orden psicológicos, vistos desde perspectivas tradicionales, aparecen como no claros y esto se evidencia en los estudios de psicología en salud ocupacional que buscan equipararlos con fenómenos naturales para poder abordarlos.

Andrade \& Gómez (2008) realizaron una revisión documental de treinta y cuatro artículos sobre salud laboral en Colombia a partir de los años noventa, encontrando un desarrollo paulatino en el área de la salud laboral que toma fuerza después del 2000. Por otro lado, plantean que a nivel académico investigativo en Colombia se encuentran en el área de salud laboral pocas investigaciones y las existentes se centran en aspectos físicos, ergonómicos y mecánicos y es a partir del 2000 que emerge el interés por temas más relacionados con lo psicológico. En las investigaciones que se interesaron en los riesgos psicosociales se encuentran principalmente problemáticas como el estrés, el burnout y el acoso laboral.

Por otro lado, el año pasado en el periódico El Espectador fue publicado un artículo en la sección de salud bajo el título "El estrés afecta al $38 \%$ de los trabajadores en Colombia" que se basa en los datos obtenidos por una empresa llamada Regus y afirma que "esta es la enfermedad que padece un gran número de trabajadores colombianos del siglo XXI" (El Espectador, 2012). Asimismo Botero (2013), presidente ejecutivo de 
la Federación de Aseguradores Colombianos (Fasecolda), refiriéndose al estrés laboral señala la dificultad para definirlo y discernir hasta qué punto es normal, es causado por factores laborales u otros factores extralaborales o la interrelación de estos factores.

Al igual que Idrovo (2003), Botero (2013) plantea la dificultad que presentan las enfermedades asociadas al trabajo a la hora de definir si las causas son de tipo laboral. Respecto a la dificultad de discernir los fenómenos psicosociales en el trabajo plantea que "en otros países, tanto como en el nuestro, empieza a notarse una tendencia a utilizar criterios laxos en la definición de la causa del estrés" (párr. 10).

Esto se evidencia en el marco de una investigación realizada sobre estrategias de intervención eficaces para la prevención y manejo del estrés laboral (Gómez, 2008), donde se entrevistaron psicólogos que trabajan de forma directa e indirecta para las diferentes aseguradoras de riesgos profesionales (ARP), ahora administradoras de riesgos laborales (ARL), y se encontró que lo que se entendía por estrés estaba asociado a procesos de adaptación y a componentes emocionales, fisiológicos, cognitivos pero que no hay elementos teóricos y conceptuales claros en los cuales se basen las intervenciones. En el caso del burnout se hace referencia a que "sí han escuchado", que "s sí saben", en algunos casos se incluyen preguntas del cuestionario de burnout en el diagnóstico del estrés, lo que muestra en la practica la dificultad de diferenciar el burnout del estrés (Díaz, 2012). Adicionalmente plantean que para las aseguradoras no es clara la diferencia y que su interés está en cumplir con los requisitos de las normas.

Frente a esta dificultad Botero (2013) revela que "el Ministerio del Trabajo está trabajando en la actualización de la tabla que recoge estas patologías y que sirve para definir las cotizaciones a cargo de los empleadores" (párr. 2) para lo cual ha contado con el apoyo de la Federación Alemana de Seguros de Accidentes, que ha compartido sus experiencias para guiar el proceso en Colombia y, a raíz de esto, se incluyó desde el 2014 en la tabla de enfermedades de origen laboral el estrés laboral y el síndrome de burnout, entre otras con el Decreto 1477 de 2014. Esto de la mano de la II Encuesta Nacional de Condiciones de Salud y de Trabajo en el Sistema General de Riesgos Laborales realizada por el Ministerio del Trabajo y la Organización Iberoamericana de Seguridad Social (OISS) que 
según la directora de Riesgos Laborales del Ministerio del Trabajo, Andrea Torres (2013) "esta herramienta fortalece las competencias del talento humano y las líneas de investigación en este tema de las universidades y la academia" (párr. 5).Como resultado de esta asesoría entre compañías de seguros surgió se expande la tabla de enfermedades laborales (Decreto 1477 de 2014) en la cual se encuentran los agentes de riesgo y el grupo de enfermedades, esto con el objetivo de facilitar la prevención y el diagnóstico médico en los trabajadores afectados. Basándose en la clasificación de la Organización Mundial de la Salud con el CIE-10, en esta nueva tabla se encuentran los agentes psicosociales y la clasificación de enfermedades. En el Grupo IV se incluyen los trastornos mentales y del comportamiento, entre los cuales se consideran la depresión, las reacciones al estrés, el trastorno del sueño, de pánico y de ansiedad y el burnout o síndrome de agotamiento profesional que figura dentro de la clasificación en el CIE-10 como "problemas relacionados con dificultades para afrontar la vida".

\section{Discusión}

En el presente apartado presentaremos algunas reflexiones que surgen de la revisión que se ha realizado haciendo énfasis en la legislación colombiana. La primera idea que se discutirá gira en torno a la problemática de seguridad y el orden social que esta implica. La segunda idea a considerar es la de la evolución conceptual desde el enfoque de los accidentes de trabajo al enfoque de los riesgos laborales como mecanismos usados en la lógica de la producción. Por último se esboza cuál es el rol de la psicología en ese contexto.

\subsection{La seguridad y el orden social desde la normativa}

Tanto la seguridad como el trabajo, la salud y la enfermedad son dimensiones que han atravesado la historia de la humanidad, relacionándose entre sí y con la configuración de la sociedad (Blanch, 2003; Guerrero \& León, 2008; Grau \& Moreno, 2000; López, Escudero \& Carmona, 2008; Rojas \& Álvarez, 2012; Yanes, 2003). 
Cuando se hace referencia a la seguridad se hace referencia a estar libre y exento de peligro; al contextualizar la seguridad en el mundo del trabajo encontramos nociones como la seguridad laboral que según Grau \& Moreno (2000) tiene diferentes connotaciones aunque realmente para ellos es un concepto único que implica que:

Las condiciones bajo las que se trabaja deben ser seguras, es decir, no deben suponer una amenaza o una posibilidad significativa de sufrir un daño de cierta entidad, que pueda incapacitar aunque sea parcial y temporalmente, por parte de los trabajadores en relación con el trabajo (p.IV.1).

Desde la Antigüedad es conocida la problemática de la relación entre el trabajo y el desarrollo de enfermedades y accidentes de trabajo, colocando en discusión la seguridad en el trabajo, la cual ha sido concebida y administrada de acuerdo a la evolución cultural, técnica, científica, sociológica, económica, legislativa y política (Arias, 2012; Henao, 2010; Novack, s.f; Melo, 2002). Sin embargo se visualiza como elemento común de la seguridad en el trabajo el hecho de que es administrada e implica un ejercicio de poder y de orden social en los diferentes momentos históricos corroborando así la premisa de Legge (1995) de que "nada cambia y sin embargo todo es diferente" (p. 385) en la relación ontológica de persona-trabajo (Rentería, 2008a; Rentería, 2008b). Por ejemplo, en el Antiguo Egipto la seguridad era suministrada por el patrón a los esclavos que construían las pirámides ya que al tener "un trato especial" realizarían su trabajo mejor, a diferencia de los esclavos de las minas, convirtiendo la seguridad desde esta época en un privilegio de unos dado por los que ejercen un poder, creando así procesos de inclusión/exclusión social.

Otro ejemplo, planteado por Schaufeli (1999), ya correspondiente a la época actual evidencia una discusión que se dio en el contexto holandés acerca de "los trabajadores menos productivos que no son capaces de afrontar las demandas laborales cada vez mayores y desarrollan quejas de salud -en especial, problemas mentales como estrés laboral severo y burnout-son empujados fuera de la población activa y reciben pensión por enfermedad o discapacidad" (p. 150). 
En Colombia esta problemática es aún más grave, puesto que sólo una minoría cuenta con un empleo formal en el cual la seguridad social permite amortiguar este tipo de situaciones. Adicionalmente debemos considerar los datos sobre empleo informal emitidos por el Departamento Administrativo Nacional de Estadística (DANE) en el cual se registra que en el segundo trimestre de 2013 un 49,6\% de los trabajadores está en condición de informalidad y, de ellos, el 59,7\% trabajan por cuenta propia; para el mismo periodo en 2014 el número de empleados particulares creció $6,2 \%$ y el de los trabajadores por cuenta propia aumentó en 1,8\%. En el 2015 el porcentaje de trabajadores informales se ubicó en $48,0 \%$ y en el tercer trimestre de 2016 la proporción de ocupados informales en las 13 ciudades y áreas metropolitanas de Colombia fue de $47,7 \%$. Otras cifras provienen de varios estudios realizados por el Banco de la República, los cuales presentan que la tasa de informalidad en Colombia, que en la mayoría de los casos corresponde a trabajadores por cuenta propia no profesionales, oscila entre $49 \%$ y $54 \%$; para mediados del 2015 el rango de informalidad estaba entre $41,8 \%$ y el 70,5\%. Estos últimos datos muestran que el comportamiento de la tasa de informalidad va en aumento en los últimos años mientras que el empleo formal ha caído (Galvis, 2012; Subgerencia Regional de Estudios Económicos de Medellín \& Unidad de Investigaciones de la Gerencia Técnica, 2015).

Situaciones semejantes viven otros países latinoamericanos en relación con la desigualdad social y los empleos informales y desprotegidos de la cobertura de la seguridad social (Díaz, 2008; Mesa-Lago, 2008). Todo esto concuerda con el planteamiento de Reygadas (2011) cuando concluye que "en México y en América Latina, donde el trabajo industrial formal, de buena calidad y con un paquete amplio de prestaciones, ha sido más la excepción que la regla" (p. 24).

El trabajo ha experimentado en las últimas décadas "profundas transformaciones tecnológicas, económicas, organizativas, espaciales, sociales, políticas y culturales" (Reygadas, 2011, p. 21). Esa transformación ha conllevado una decadencia de las formas tradicionales del trabajo y, por el otro lado, un aumento de otras actividades laborales, como la actividad intelectual, la prestación de servicios, el trabajo informal, la flexibilización del empleo y los trabajos atípicos. Si bien Rafael Pardo, ministro de Trabajo entre 2011 y 2014, planteó que con la Ley 1562 de 2012 se tenga 
como meta que "ni un solo trabajador en Colombia esté por fuera del sistema de protección social", intentando incluir otras formas de trabajo como el de los trabajadores independientes con contrato superior a un mes, quienes deberán cotizar de manera obligatoria con el nuevo Sistema General de Riesgos Laborales, la nueva ley plantea, en cuanto a los trabajadores informales, que éstos podrán afiliarse voluntariamente. Esto muestra claramente nuevas formas de orden social, donde los trabajadores con unas condiciones de trabajo más precarias, con menores ingresos y afectados por la desigualdad y la pobreza como los trabajadores informales (Freije, 2001) no son protegidos y deben ellos mismos asumir su seguridad, colocando así la responsabilidad en el sujeto trabajador (Beck E Beck-Gernshein, 2002; Rentería, 2016; Díaz, 2013), exonerando a las organizaciones y al Estado, desplazando así la seguridad social por la seguridad personal (Bauman, 2007).

Esto también es claro en el último discurso de Botero en la Convención Internacional de Seguros en septiembre de 2015, donde plantea que "el Sistema de Riesgos Laborales es el otro módulo de la Seguridad Social que ha sido encomendado a compañías de seguros" (p. 6) y donde a su vez plantea que una de las principales preocupaciones es que la tendencia a que:

Los mayores riesgos y los que generan mayor carga operativa se concentren en ciertas compañías, en especial la de origen estatal, ha ordenado que el conjunto de ellas suscriban un acuerdo para distribuir los afiliados que por razones de alta siniestralidad o costo se desvíen respecto de una normalidad hipotética. Como no fue posible, a pesar de persistentes esfuerzos, llegar a ese compromiso, el Gobierno se encuentra próximo a definir el esquema de compensación (p. 6).

\subsection{La evolución conceptual desde el enfoque de los accidentes de trabajo al de los riesgos laborales como mecanismos usados en la lógica de la producción}

El siguiente aspecto a tratar está relacionado con los accidentes y los riesgos en el campo de la salud ocupacional. Si al inicio de las regulaciones de seguridad en el trabajo en América Latina estaban relacionadas con los accidentes de trabajo (Bronstein, 1998; García, 2008), Colombia 
no fue la excepción. Con el presidente Rafael Uribe Uribe y con la Ley 57 de 1915 se regula la seguridad haciendo énfasis en la reparación por accidentes de trabajo. En la actualidad Colombia tiene la Ley 1562 de 2012 que hace énfasis en el Sistema General de Riesgos Laborales. Es importante dejar claro que una categoría no excluye la otra: la diferencia radica en donde se pone el énfasis.

El concepto de accidente de trabajo a lo largo del desarrollo legislativo en Colombia ha estado atravesado por varios elementos a considerar. Uno de ellos es la culpa, el otro el hecho de ser un evento imprevisto y de fuerza mayor (ver Tabla 1). Conceptos como la culpa traen consigo la idea que hay un acto por parte del trabajador que debería ser previsto, o que fue causado por una imprudencia o por una falta, en lugar de considerarlo como un evento imprevisto y de fuerza mayor que implica un hecho no controlable. Ayala (2001) define un suceso repentino como "el acontecimiento cierto y real que se presenta de pronto, de manera instantánea y que obra durante un periodo breve sobre el organismo del trabajador, aunque las consecuencias se manifiesten posteriormente" (p. 117). En concordancia con esto

Virilio (2009) plantea que existen accidentes naturales inesperados y accidentes artificiales que están asociados con los avances tecnológicos llevando a una industrialización del accidente artificial con lo cual "el accidente ya no es más inesperado [...] en el supuesto de la culpa tiende a prevalecer sobre el de lo involuntario" (p. 33). Esto trae nuevamente la discusión sobre la responsabilización de los trabajadores puesto que, al no poder culpar al patrono/empleador el trabajador no tenía derecho a la reparación o a la indemnización. Esto cambia con el Decreto 1295 de 1994. Se retira el concepto de culpa que desvía la atención del origen laboral del suceso y así es a partir de las nuevas reglamentaciones donde la culpa del trabajador no es un hecho que lo excluye del origen laboral.

El riesgo como otro elemento importante en la psicología de la salud ocupacional y en la salud laboral se plantea ya desde el Código Sustantivo del Trabajo de 1950, pero relacionado con el accidente de trabajo. Como categoría central empieza a emerger el riesgo a partir de la década de los noventa. Es así como encontramos en Colombia un cambio de énfasis en la legislación del accidente de trabajo al riesgo. En el Decreto 614 de 1984 se halla una de las primeras definiciones de riesgo en la legisla- 
ción colombiana. Además plantea allí que uno los objetivos de la salud ocupacional es proteger a la persona contra los riesgos relacionados con agentes físicos, químicos, biológicos, psicosociales, mecánicos, eléctricos y otros derivados de la organización laboral que puedan afectar la salud individual o colectiva en los lugares de trabajo.

Si bien en las normativas se utiliza con alta frecuencia el término, solo se encuentran tres definiciones (ver Tabla 2). En estas podemos ver dos elementos importantes cuando se habla de riesgo, uno es el carácter latente, permanente, "presente" posible y el otro gira en torno al daño, acorde con la definición "de riesgo como la posibilidad de que ocurra un daño" (Vargas, 2004, p. 35). El riesgo implica la previsión y el control del futuro, de la posibilidad que no es un hecho en sí, es decir alude a una destrucción pero no es la destrucción en sí (Beck, 2002). Por su parte, Bauman (2010) plantea que "los riesgos son aquellos peligros cuya probabilidad podemos (o creemos ser capaces de) calcular: los riesgos

Tabla 1. Definiciones de accidente de trabajo en la legislación colombiana

\begin{tabular}{|c|c|c|c|c|}
\hline Ley 57 de 1915 & Ley 6 de 1945 & $\begin{array}{l}\text { Código Sustantivo } \\
\text { del Trabajo en } 1950\end{array}$ & $\begin{array}{l}\text { Decreto } 1295 \\
\text { de } 1994\end{array}$ & $\begin{array}{l}\text { Ley } 1562 \\
\text { de } 2012\end{array}$ \\
\hline $\begin{array}{l}\text { Artículo } 1^{\circ} \\
\text { enriéndese } \\
\text { por accidente } \\
\text { de trabajo } \\
\text { un suceso } \\
\text { imprevisto } \\
\text { y repentino } \\
\text { sobrevenido } \\
\text { por causa y } \\
\text { con ocasión del } \\
\text { trabajo, y que } \\
\text { produce en el } \\
\text { organismo de } \\
\text { quien ejecuta } \\
\text { un trabajo por } \\
\text { cuenta ajena } \\
\text { una lesión o una } \\
\text { perturbación } \\
\text { funcional } \\
\text { permanente o } \\
\text { pasajera, todo } \\
\text { sin culpa del } \\
\text { obrero. }\end{array}$ & $\begin{array}{l}\text { Artículo } 12 .^{\circ} \text { Para } \\
\text { estos efectos se } \\
\text { entiende por } \\
\text { accidentes de trabajo } \\
\text { toda lesión orgánica } \\
\text { o perturbación } \\
\text { funcional que afecte } \\
\text { al trabajador en } \\
\text { forma transitoria, } \\
\text { permanente } \\
\text { o definitiva, } \\
\text { motivada por un } \\
\text { hecho imprevisto } \\
\text { y repentino, que } \\
\text { sobrevenga por } \\
\text { causa o con ocasión } \\
\text { del trabajo, siempre } \\
\text { que la lesión o } \\
\text { perturbación no } \\
\text { sea provocada } \\
\text { deliberadamente, } \\
\text { o por falta grave o } \\
\text { intencional de la } \\
\text { víctima. }\end{array}$ & $\begin{array}{l}\text { Artículo } 1^{\circ} \text { Se } \\
\text { entiende por } \\
\text { accidente de } \\
\text { trabajo todo } \\
\text { suceso imprevisto } \\
\text { y repentino } \\
\text { que sobrevenga } \\
\text { por causa o } \\
\text { con ocasión del } \\
\text { trabajo en una } \\
\text { lesión orgánica } \\
\text { o perturbación } \\
\text { funcional } \\
\text { permanente } \\
\text { o pasajera, y } \\
\text { que no haya } \\
\text { sido provocado } \\
\text { deliberadamente o } \\
\text { por culpa grave de } \\
\text { la víctima. }\end{array}$ & $\begin{array}{l}\text { Artículo 9. } \\
\text { Es accidente } \\
\text { de trabajo } \\
\text { todo suceso } \\
\text { repentino que } \\
\text { sobrevenga } \\
\text { por causa o } \\
\text { con ocasión } \\
\text { del trabajo, } \\
\text { y que } \\
\text { produzca en } \\
\text { el trabajador } \\
\text { una lesión } \\
\text { orgánica, una } \\
\text { perturbación } \\
\text { funcional, una } \\
\text { invalidez o la } \\
\text { muerte. }\end{array}$ & $\begin{array}{l}\text { Artículo } 3 .^{\circ} \\
\text { Es accidente } \\
\text { de trabajo } \\
\text { todo suceso } \\
\text { repentino que } \\
\text { sobrevenga } \\
\text { por causa o } \\
\text { con ocasión } \\
\text { del trabajo, } \\
\text { y que } \\
\text { produzca en } \\
\text { el trabajador } \\
\text { una lesión } \\
\text { orgánica, una } \\
\text { perturbación } \\
\text { funcional o } \\
\text { psiquiátrica, } \\
\text { una invalidez } \\
\text { o la muerte. }\end{array}$ \\
\hline
\end{tabular}

Fuente: elaboración propia 
son lo más parecido que podemos tener a la (por desgracia inalcanzable) certeza" (p. 20).

Beck (2000) explica que el discurso del riesgo se da cuando la confianza en la seguridad termina. Eso tiene una clara relación con el tránsito de la seguridad social a la seguridad personal (Bauman, 2010). Beck (2000) afirma:

El discurso del riesgo empieza donde la confianza en nuestra seguridad termina, y deja de ser relevante cuando ocurre la potencial catástrofe. El concepto de riesgo delimita, por tanto, un peculiar estado intermedio entre seguridad y destrucción, donde la percepción de riesgos amenazantes determina pensamiento y acción (p. 10).

Tabla 2. Definiciones de riesgo en la legislación colombiana

\begin{tabular}{|l|l|l|l|}
\hline \multicolumn{1}{|c|}{$\begin{array}{c}\text { Decreto 614 } \\
\text { de 1984 }\end{array}$} & \multicolumn{1}{|c|}{ Decreto 1295 de 1994 } & $\begin{array}{c}\text { Resolución 2646 } \\
\text { de 2008 }\end{array}$ & \multicolumn{1}{c|}{$\begin{array}{c}\text { Decreto 1443 } \\
\text { de 2014 }\end{array}$} \\
\hline $\begin{array}{l}\text { Riesgo potencial: } \\
\text { Es el riesgo de } \\
\text { carácter latente, } \\
\text { susceptible de } \\
\text { causar daño a la } \\
\text { salud cuando fallan } \\
\text { o dejan de operar } \\
\text { los mecanismos de } \\
\text { control. }\end{array}$ & $\begin{array}{l}\text { Riesgos Profesionales. Son } \\
\text { Riesgos Profesionales el } \\
\text { accidente que se produce } \\
\text { como consecuencia } \\
\text { directa del trabajo o } \\
\text { labor desempeñada, y la } \\
\text { enfermedad que haya sido } \\
\text { catalogada como profesional } \\
\text { por el Gobierno Nacional. }\end{array}$ & $\begin{array}{l}\text { Riesgo: } \\
\text { Probabilidad de } \\
\text { ocurrencia de una } \\
\text { enfermedad, lesión } \\
\text { o daño en un } \\
\text { grupo dado. }\end{array}$ & $\begin{array}{l}\text { Combinación de } \\
\text { la probabilidad de } \\
\text { que ocurra una o } \\
\text { más exposiciones o } \\
\text { eventos peligrosos } \\
\text { y la severidad del } \\
\text { daño que puede ser } \\
\text { causada por estos. }\end{array}$ \\
\hline
\end{tabular}

Fuente: elaboración propia

¿Qué implica poner el énfasis en el riesgo? Cuando se concibe la vida en el trabajo desde el riesgo se pone de frente la incertidumbre, el ser susceptibles al daño, al peligro; allí emerge la inseguridad, la vulnerabilidad. Bauman (2010) plantea tres clases de peligros a los cuales se les teme: el primero es la amenaza al cuerpo y las propiedades de las personas; el segundo peligro al cual hace referencia es la seguridad de los medios de vida como el empleo y la renta o la supervivencia en el caso de invalidez o vejez; el tercer peligro está relacionado con el lugar de la persona en el mundo, su posición jerárquica e identidad. Estos peligros generan el sentimiento de miedo, en el cual diferencia dos niveles, uno que es compartido por todas las criaturas vivas y el segundo, el miedo derivativo (sentimiento de ser susceptible) que orienta la conducta sea 
que haya o no una amenaza inmediatamente presente. De esta manera pone en acción la "capacidad autopropulsora" (Bauman, 2010, p. 12) o en términos de Beck (2000) "los riesgos en los que se cree son el látigo empleado para mantener el momento presente corriendo a galope" (p. 12).

Esto en el contexto del trabajo se logra a través de la administración o gestión del miedo (Dejours, 2009; Virilio, 2009). Un ejemplo de esto es presentado por Vesga (2007) en una investigación sobre tipos de contratación laboral y el contrato psicológico en Colombia encontrando que:

En las formas flexibles de contratación como son el outsourcing y las CTA, los trabajadores por sentirse más vulnerables al no contar con una estabilidad laboral, tienden a construir un contrato psicológico con compromisos de sí mismos que comprenden conductas de ciudadanía organizacional o la intención de hacer tareas adicionales como estrategias para tener la posibilidad de vincularse como empleados a la organización (p. 180).

En este mismo sentido, Dejours (2009) plantea sobre la gestión del miedo "esgrimiendo la amenaza de despido, pueden conseguir lo que los operadores aumenten su ritmo de trabajo más de lo que se creía posible" (p. 76). Esto permite la disciplinarización del trabajador (Spink, 2003), la aceptación de la injusticia y la precarización (Dejours, 2009) y, con ello, la perpetuación de las lógicas de producción.

Las concepciones de salud en el trabajo inicialmente se centraban en accidentes de trabajo, en las lesiones y daños de orden físico, esto se ve en primeras legislaciones. Con los cambios en las formas de trabajo la preocupación cambia por la salud mental en el mundo. Es aquí donde la psicología ha tenido un papel protagónico desde comienzos del siglo pasado con la fatiga y ahora con el estrés, el acoso y el burnout.

\subsection{La Psicología en la normativa}

En Colombia, a partir del Decreto 614 de 1984, comienza a aparecer el sufijo psi- o la referencia a lo psicológico a nivel de los riesgo y, con esto, se abre paso el aspecto del riesgo psicológico dos décadas después a la Resolución 2646 de 2008 cuando se define el riesgo (ver Tabla 2) 
y esto va acompañado de la noción de factores de riesgo psicosocial en la cual no queda especificado qué es lo psicosocial. Actualmente se presenta el Decreto 1477 de 2014 en el cual ya se tiene dentro de la tabla de enfermedades de origen laboral un apartado para los trastornos mentales y del comportamiento con diecisiete posibles patologías entre las cuales se encuentran el estrés y el burnout.

En disonancia con ello desde la Resolución 2646 se define como experto en riesgo psicosociales al "Psicólogo con posgrado en salud ocupacional, con licencia vigente de prestación de servicios en psicología ocupacional" (Artículo 3. ${ }^{\circ}$ ), lo cual ha dado un nuevo campo laboral y de estudio de postgrado a los psicólogos en Colombia.

Este contexto, junto con la idealización de la ciencia y del conocimiento psicológico, conllevó a la consolidación de la psicología de la salud ocupacional, en coherencia con la idea de Illich (1981) sobre una "era de las profesiones inhabilitantes" la cual implica que las personas tienen "problemas", los expertos tienen "soluciones" ya que "hoy los doctores y los asistentes sociales - como antes sólo lo hacían los sacerdotes y los juristas - consiguen poder legal para crear la necesidad que, por ley, únicamente ellos están autorizados a satisfacer" (párr. 10). De esta forma, estos profesionales promulgan tener un conocimiento que solo ellos tendrán derecho a administrar.

Con la administración de los riesgos se controla el trabajo. En este sentido Pulido \& Carvajal (2013) plantean que "la psicología se presenta alrededor del manejo del riesgo en relación con la producción de la subjetividad de los trabajadores contemporáneo" (p. 70) Frente a esta situación es interesante el siguiente dato: la segunda Encuesta europea de empresas sobre riesgos nuevos y emergentes (ESENER-2) realizada por la Agencia Europea para la Seguridad y la Salud en el Trabajo (2014) demuestra que en los países de la Unión Europa en el abordaje de los riesgo psicosociales se hace uso de psicólogos solo en el 16\% de un total de 49320 empresas. Esto hace reflexionar si esta hiperespecialización del campo de trabajo y del conocimiento realmente conduce al mejoramiento de las condiciones de trabajo y a la disminución de los riesgo psicosociales o sería mejor una ampliación que permita una lectura más enriquecida y amplia sobre estas problemáticas donde la única voz no sea la del experto. 


\section{Conclusiones}

Se concluye que cuando se hace el recorrido histórico sobre las repercusiones del trabajo en la salud es necesario remitirse hasta la Antigüedad, mostrando que, si bien ahora es un campo relativamente joven y en la psicología más aún, la relación existente entre el trabajo y la salud ha sido y hace parte de la relación ontológica de persona-trabajo a lo largo de la historia. Adicionalmente, se logra visualizar como en diferentes momentos la seguridad en el trabajo trae consigo un ejercicio de poder y de orden social que, a través de diversos mecanismos como la gestión del miedo, permiten la disciplinarización, la precarización y la administración a los trabajadores.

El ejercicio de los psicólogos en el campo de la salud en el trabajo se ha normativizado y tecnificado en la última década, con esto se ha habilitado a aquellos que han institucionalizado su saber y, por lo tanto, están licenciados para aplicar los procedimientos y recurso de la psicología en salud ocupacional, sin tener claro si esto contribuye claramente al mejoramiento de las condiciones de trabajo y a la disminución de los riesgos psicosociales.

Estas reflexiones hacen surgir cuestionamientos que serán necesarios seguir desarrollando en este campo, considerando que en Colombia se han generado nuevos decretos que pueden conllevar una mayor patologización en el país: ¿Cómo trabajar a favor de la emancipación de los trabajadores desde la salud y la psicología sin perpetuar las lógicas capital/trabajo que han atravesado los diferentes abordajes en este campo?, ¿será posible mejorar las contradicciones de trabajo-riesgo-salud?, ¿cuál es el papel de los psicólogos colombianos en la construcción de las normativas que regulan su ejercicio? y ¿cómo construir diálogos reales entre la académica, la investigación, los legisladores y ejecutantes de la ley y los trabajadores que permitan construir otras formas de comprender e intervenir la salud en el trabajo? 


\section{Referencias}

Agencia Europea para la Seguridad y la Salud en el Trabajo. (2014). "Segunda Encuesta Europea de Empresas sobre Riesgos Nuevos y Emergentes (ESENER-2)". Encuesta. Recuperado de https://osha.europa.eu/es/surveys-andstatistics-osh/esener

Andrade, V., E Gómez, I. (2008). Salud laboral. Investigaciones realizadas en Colombia. Pensamiento Psicológico, 4(10), 9-25.

Arana, R., E Guerrero, I. (2013). Movimiento obrero y protesta social en Colombia. 1920-1950. Historia Caribe, 8(22), 167-193.

Arias, W. (2012). Revisión histórica de la salud ocupacional y la seguridad industrial. Revista Cubana de Salud y Trabajo, 13(3), 45-52.

Ayala, C. (2001). Legislación en Salud Ocupacional y Riesgos Profesionales. Bogotá: Ediciones Salud Laboral.

Bauman, Z. (2007). Miedo líquido: La sociedad contemporánea y sus temores. España: Paidós.

Beck, U. (2000). Retorno a la teoría de la "sociedad del riesgo". Boletín de la A.G.E, 30, 9-20.

Beck, U. (2002). La Sociedad del Riesgo Global. Madrid: Siglo XXI.

Beck, U., E Beck-Gernshein, E. (2002). Individualization. Londres: Sage.

Beltrán, O. (2005). Revisiones sistemáticas de literatura. Revista Colombiana de Gastroenterología, 20(1), 60-69.

Blanch, J. (2003). Trabajar en la modernidad industrial. En J. Blanch (Coord.), Teoría de las Relaciones Laborales (pp. 19-148). Barcelona: UOC.

Botero, J. (16 de noviembre de 2013). Estrés laboral. Portafolio. Recuperado de http://www.portafolio.co/opinion/jorge-humberto-botero-1/estreslaboral-74620

Botero, J. (2015). Solidaridad social: eje de la actividad aseguradora. Recuperado de http://www.fasecolda.com/files/6714/1217/discurso_Inauguracin-_JOrgeH-Botero-CVN15.

Bronstein, A. (1998). Pasado y presente de la legislación laboral en América Latina. Recuperado de http://www.ilo.org/public/spanish/region/ampro/ mdtsanjose/papers/pasado

Cadavid, T. (1942). Raíces griegas y latinas: etimologías médicas y biológicas. Colombia: Editorial de la Litografía Colombiana.

Colombia, Congreso de Colombia. Ley 57 de 1915 (15 de noviembre de 1915).

Colombia, Congreso de Colombia. Ley 46 de 1918 (19 de noviembre de 1918). 
Colombia, Congreso de Colombia. Ley 96 de 1938 (6 de agosto de 1938).

Colombia, Congreso de Colombia. Ley 6 de 1945 (19 de febrero de 1945).

Colombia, Congreso de Colombia. Ley 90 de 1946 (26 de diciembre de 1946).

Colombia, Congreso de Colombia. Ley 9 de 1979 (24 de enero de 1979).

Colombia, Congreso de Colombia. Ley 100 de 1993 (23 de diciembre de 1993).

Colombia, Congreso de Colombia. Ley 1010 de 2006 (23 de enero de 2006).

Colombia, Congreso de Colombia. Ley 1562 de 2012 (11 de julio de 2012).

Colombia, Ministerio de Trabajo \& Ministerio de Salud y Seguridad Social. Resolución 1016 de 1989 (31 de marzo de 1989).

Colombia, Ministerio de la Protección Social. Resolución 002646 de 2008 (17 de julio de 2008).

Colombia, Presidencia de la República. Decreto 586 de 1983 Por el cual se crea el "Comité de salud Ocupacional (17 de marzo de 1983).

Colombia, Presidencia de la República. Decreto 614 de 1984 Por el cual se determinan las bases para la organización y administración de Salud Ocupacional en el país (14 de marzo de 1984).

Colombia, Presidencia de la República. Decreto 1295 de 1994 Por el cual se determina la organización y administración del Sistema General de Riesgos Profesionales (22 de junio de 1994).

Colombia, Presidencia de la República. Decreto 1477 de 2014 Por el cual se expide la Tabla de Enfermedades Laboral (5 de agosto de 2014).

Cruz, A. (1996). Grandes Oradores Colombianos. Bogotá: Imprenta Nacional de Colombia.

Dejours, C. (2009). Trabajo y sufrimiento. Cuando la injusticia se hace banal. Madrid: Modus Laborandi.

Departamento Administrativo Nacional de Estadística. (2013). Resumen ejecutivo sobre medición del empleo informal y seguridad social, Trimestre móvil junio - agosto de 2013. Recuperado de http://www.dane.gov.co/files/investigaciones/ boletines/ech/ech_informalidad/re_ech_informalidad_jun_ago2013.pdf

Departamento Administrativo Nacional de Estadística. (2014). Principales indicadores del mercado laboral. Septiembre de 2014. Recuperado de http://www. dane.gov.co/files/investigaciones/boletines/ech/ech/bol_empleo_sep_14.pdf

Departamento Administrativo Nacional de Estadística. (2016). Boletín técnico sobre medición del empleo informal y seguridad social, Trimestre julio - septiembre de 2016. Recuperado de https://www.dane.gov.co/files/investigaciones/ boletines/ech/ech_informalidad/bol_ech_informalidad_jul_sep16.pdf 
Díaz, F. (2012). Sindrome de burnout: desarrollos conceptuales y empíricos. (Disertación de maestría en psicología, Universidad del Valle, Cali, Colombia). Universidad Del Valle. Colombia.

Díaz, F. (2013). Burnout. Una metáfora para comprender relaciones y contextos de trabajo desde la salud. (Proyecto de tesis doctoral en psicología). Universidad del Valle, Cali, Colombia.

Díaz, L. E. (2008). Cobertura previsional de los trabajadores independientes. Estudios Socio-Jurídicos, 10(2), 37-75.

Elías, J. (2013). La masacre obrera de 1928 en la zona bananera del Magdalena-Colombia. Una historia inconclusa. Andes (Salta), 22(1). Recuperado de http://www.scielo.org.ar/scielo.php?script=sci_arttextEpid=S166880902011000100004 \&lng=es\&tlng=es

Estrés afecta al 38\% de los trabajadores en Colombia (7 de septiembre de 2012). El Espectador. Recuperado de http://www.elespectador.com/impreso/salud/ articulo-373408-estres-afecta-al-38-de-los-trabajadores-colombia

Freije, S. (2001). El Empleo Informal en América Latina y el Caribe: Causas, consecuencias y recomendaciones.. Recuperado de http://www.iadb.org/wmsfiles/ products/publications/documents/354774.pdf

Galvis, A. (2012). Informalidad laboral en las áreas urbanas de Colombia. Cartagena: Banco de la Republica. Recuperado de http://www.banrep.gov.co/sites/ default/files/publicaciones/archivos/DTSER-164.pdf

García, A. (2008). El Sistema General De Riesgos Profesionales vigente en Colombia - una visión interna y desde la decisión 584, instrumento andino de seguridad y salud en el trabajo-. International Law Revista Colombiana de Derecho Internacional, 13, 215-253.

Gómez, I. (2008). Estrategias de intervención eficaces para la prevención y manejo del estrés laboral. (Manuscrito no publicado). Universidad del Valle, Cali.

Guerrero, L., E León, A. (2008). Aproximación al concepto de salud. Revisión histórica. Fermentum. Revista Venezolana de Sociología y Antropología, 18(53), 610-633.

Grau, M., E Moreno, D. (2000). Seguridad laboral [Versión Adobe Digital Editions]. Recuperado de http://www.bvsde.paho.org/bvsacd/cd46/LSI_Cap04.pdf Henao, F. (2010). Salud Ocupacional: conceptos básicos (2. edición). Colombia: Ecoe Ediciones.

Idrovo, A. (2003). Estimación de la incidencia de enfermedades ocupacionales en Colombia, 1985-2000. Revista de Salud Pública, 5, 263-71. 
Illich, I. (1981). Las profesiones inhabilitantes. En Desactivación Profesiones. Madrid: Herman Blume Ediciones. Recuperado de https://es.scribd.com/ doc/187776401/Profesiones-inhabilitantes-Ivan-Illich-pdf

Legge, K. (1 995). Human Resources Management. Reino Unido: Macmillan Education UK.

Letelier, L.M., Manríquez, M., Juan, J., E Rada, G. (2005). Revisiones sistemáticas y meta-análisis: ¿son la mejor evidencia? Revista Médica de Chile, 133(2), 246-249.López, O., Escudero, J., \& Carmona, L. (2008). Los determinantes sociales de la salud. Una perspectiva desde el Taller Latinoamericano de Determinantes Sociales de la Salud, ALAMES. Medicina Social, 3(4), 323-335.

Matabanchoy, S. (2012). Salud en el trabajo. Universidad y Salud, 14(1), 87-102.

Mesa-Lago, C. (2008). Un reto de Iberoamérica en el siglo XXI: la extensión de la cobertura de la Seguridad Social. América Latina Hoy, 48, 67-81.

Melo, J. (7 de julio de 2002). Historia de la ergonomía. Estrucplan.Recuperado de http://www.estrucplan.com.ar/producciones/entrega.asp?identrega=55

Ministerio de la Protección Social (2007). "Primera Encuesta Nacional de Condiciones de Salud y Trabajo en el Sistema General de Riesgos Profesionales (I ENCST)" Encuesta. Recuperado de http://www.oiss.org/estrategia/IMG/ pdf/I_encuesta_nacional_colombia2.pdf

Ministerio de la Protección Social (2010). Batería de instrumentos para la evaluación de factores de riesgo psicosocial. Bogotá: Ministerio de la Protección Social.

Ministerio de la Protección Social (2013). "Segunda Encuesta Nacional de Condiciones de Seguridad y Salud en el Trabajo en el Sistema General de Riesgos Laborales de Colombia (II ENCSST)" Encuesta. Recuperado de http://ccs. org.co/salaprensa/images/Documentos/INFORME_EJECUTIVO_II\%20 ENCSST.pdf

Nelson, A.M. (2002). A metasynthesis: Mother in go ther-than-normal children. Qualitative Health Research, 12(4), 515-530.

Novack, G. (sin fecha). La Primera Internacional (1864-76). Recuperado de http://www.marxists.org/espanol/novack/1977/1 inter.htm

Perestelo-Pérez, L. (2013). Standards on how to develop and report systematic reviews in Psychology and Health. International Journal of Clinical and Health Psychology, 13(1), 49-57.

Pulido, H. \& Carvajal, L. (2013). Riesgo psico-laborales y subjetividad: tensiones contemporáneas entre patologización y despatologización en los discursos psicológicos. Quaderns de psicología, 15(1), 69-80.

Rentería, E. (2008a). Empregabilidad: versões e implicações. Uma Leitura desde a Psicología social. (Tesis de doctorado, Universidad de São Paulo, São Paulo, 
Brasil). Recuperado de http://www.teses.usp.br/teses/disponiveis/47/47134/ tde-12022009-143157/pt-br.php

Rentería, E. (2008b) Nuevas realidades organizacionales y del mundo del trabajo: Implicaciones para la construcción de la identidad o del sujeto. Informes Psicológicos, 10, 65-80.

Rentería, E. (2016) Salud, bienestar y mundo del trabajo. Entre lo normativo, los riesgos, y la coexistencia de realidades. En M. Costa., \& J. Zanatta. (Orgs.), Psicología da Saúde: discussões temáticas (pp. 171-192). Campo Grande: Editora da UCDB.Rojas, F., E Álvarez, A. (2012). Seguridad Humana. Un estado del arte. En F. Rojas, (Ed.), Seguridad humana, nuevos enfoques (9-32). San José: FLACSO.

Reygadas, L. (2011). Introducción. Trabajos atípicos, trabajos precarios: ¿dos caras de la misma moneda? En E. Pacheco, E. de la Garza, E L. Reygadas (Coords.) Trabajos atípicos y precarización del empleo. México: El Colegio de México.

Schaufeli, W. (1999). Evaluación de riesgo psicosociales y prevención del estrés laboral: algunas experiencias holandesas. Revista de psicología del trabajo y de las Organizaciones, 15(82), 141-171.

Spink, M. J. (2003). Psicologia social e saúde; práticas, saberes e sentidos. Petrópolis: Editora Vozes Ltda.

Subgerencia Regional de Estudios Económicos de Medellín \& Unidad de Investigaciones de la Gerencia Técnica (2015). La informalidad en el mercado laboral colombiano. Recuperado de http://www.banrep.gov.co/sites/default/ files/publicaciones/archivos/re_197.pdf

Toro, A.G. (2001). Enfermería basada en la evidencia: cómo incorporar la investigación a la práctica de los cuidados. Granada: Fundación Index. Recuperado de http://www.index-f.com/evidentia/clasificacion_evid_cualiativas.php

Vargas, C. (2004). Análisis epistemológico del riesgo. Revista de Filosofía del Universidad de Costa Rica, XLII(106-107), 35-42.

Vega, R. (2002). Gente muy rebelde: protesta popular y modernización capitalista en Colombia (1909-1929) (1. ' Volumen). Bogotá: Ediciones Pensamiento Crítico.

Vesga, J. (2011). Los tipos de contratación laboral y sus implicaciones en el contrato psicológico. Pensamiento Psicológico, 9(6), 171-181. Recuperado de http://www.umanizales.edu.co/publicaciones/campos/sociales/perspectivas_psicologia/html/revistas_contenido/revista 10/Contenidodelcontrato.pdf Virilio, P. (2009). El accidente original. Buenos Aires: Amorrortu.

Yanes, L. (2003). El trabajo como determinante de la salud. Salud de los Trabajadores, 11(1), 21-4. 